ARTICLE

https://doi.org/10.1038/s41467-019-11706-8

\title{
Atomic structure of the Epstein-Barr virus portal
}

\author{
Cristina Machón (10) 1,2, Montserrat Fàbrega-Ferrer ${ }^{1,2}$, Daming Zhou (ib ${ }^{3}$, Ana Cuervo ${ }^{4}$, José L. Carrascosa ${ }^{4}$, \\ David I. Stuart (iD ${ }^{3} \&$ Miquel Coll (1D ${ }^{1,2}$
}

Herpesviridae is a vast family of enveloped DNA viruses that includes eight distinct human pathogens, responsible for diseases that range from almost asymptomatic to severe and lifethreatening. Epstein-Barr virus infects B-cells and epithelial cells, causing infectious mononucleosis, as well as a number of cancers. Epstein-Barr infection cannot be cured since neither vaccine nor antiviral drug treatments are available. All herpesviruses contain a linear double-stranded DNA genome, enclosed within an icosahedral capsid. Viral portal protein plays a key role in the procapsid assembly and DNA packaging. The portal is the entrance and exit pore for the viral genome, making it an attractive pharmacological target for the development of new antivirals. Here we present the atomic structure of the portal protein of Epstein-Barr virus, solved by cryo-electron microscopy at $3.5 \AA$ resolution. The detailed architecture of this protein suggests that it plays a functional role in DNA retention during packaging.

\footnotetext{
${ }^{1}$ Institute for Research in Biomedicine (IRB Barcelona), The Barcelona Institute of Science and Technology (BIST), Baldiri Reixac 10-12, 08028 Barcelona, Spain. ${ }^{2}$ Molecular Biology Institute of Barcelona (IBMB-CSIC), Baldiri Reixac 10-12, 08028 Barcelona, Spain. ${ }^{3}$ Division of Structural Biology, University of Oxford, The Henry Wellcome Building for Genomic Medicine, Headington, Oxford OX3 7BN, UK. ${ }^{4}$ Department of Macromolecular Structures, Centro Nacional de Biotecnología-Consejo Superior de Investigaciones Científicas (CNB-CSIC), 28049 Madrid, Spain. Correspondence and requests for materials should be addressed to M.C. (email: miquel.coll@irbbarcelona.org)
} 
$\mathrm{T}$ he eight herpesviruses infecting humans cause different disorders and are classified into three subfamilies, namely $\alpha, \beta$, and $\gamma$. Alphaherpesvirinae include herpes simplex virus types 1 and 2 (HSV-1, HSV-2), and varicella-zoster virus (VZV), causing herpes labialis/genitalis and chickenpox, respectively. Betaherpesvirinae include cytomegalovirus (CMV) and roseolovirus (HHV-6, HHV-7), which cause infectious mononucleosis and roseola infantum, respectively. Finally, gammaherpesvirinae, which include Kaposi's sarcoma-associated virus (KSHV) and Epstein-Barr virus (EBV) are associated with different types of cancer. EBV causes infectious mononucleosis, a number of cancers, such as Burkitt's, Hodgkin's and T-cells lymphomas, and gastric and naseopharyngeal carcinomas and several autoimmune diseases such as lupus erythematosus ${ }^{1-3}$.

All herpesviruses have a portal pore for capsid assembly and DNA packaging and ejection. On the basis of their capsid structure and viral DNA packaging mechanism, it has been suggested that herpesviruses and tailed bacteriophages are evolutionarily related ${ }^{4-6}$. Portal proteins are located at a unique capsid vertex and are one of the conserved elements within herpesvirus and bacteriophages, even though there is no sequence similarity between them, nor among the different bacteriophage portals solved. In early stages of infection, portal proteins are involved in the release of the viral genome from the capsid to the cell. Transcription of the viral genes, together with extensive replication of long concatameric DNA, takes place once a lytic infection is triggered. Later in the infection, portal proteins are among the first proteins recruited during the assembly of the new procapsids. In addition, they interact with the terminase complex, which provides the energy and force necessary for packaging the DNA inside the capsid and cleaves the DNA at the appropriate site. In bacteriophages, portal proteins also serve as a connection between the capsid and the tail proteins ${ }^{7,8}$. Given their key function in DNA packaging during viral replication', portal proteins are attractive targets for the development of antiviral drugs. However, high-resolution atomic structures are not available for structure-driven drug design that specifically targets the portal.

The structure determination at high resolution of portal proteins from bacteriophages has been successful with examples of gp6 from SPP1, gp1 from P22, gp20 from T4, and gp10 from $\phi 29$, solved by X-ray crystallography and cryo-EM ${ }^{10-14}$. However, the study of herpesvirus portals has been more challenging because of the difficulty in expressing them in soluble form and in obtaining crystals. This difficulty is likely attributable to a large flexible area in their structure (see below). Consequently, to date, only limited structural studies of herpesvirus portals have been performed, all at low resolution ${ }^{15-18}$. While we were submitting this paper, a cryo-EM structure of the portal vertex of HSV-1, an alpha herpesvirus, was published. The structure was solved by symmetry relaxation of the whole virion, with the portal determined at $5.6 \AA$ resolution, although coordinates and maps were not yet available for precise comparison with our EBV portal ${ }^{19}$. Here we present the atomic structure of the EBV portal solved by cryo-electron microscopy at $3.5 \AA$ resolution, showing its detailed architectural features.

\section{Results}

The putative portal protein of EBV, pBBRF1, which has an expected molecular weight of $68 \mathrm{kDa}$ as a monomer, was expressed in insect cells and purified as a fusion protein with a His-Z-tag in four chromatography steps, in the presence of $\mathrm{n}$ dodecyl $\beta$-D-maltoside to increase its stability. The quality and homogeneity of the sample was tested by negative-stain electron microscopy (data not shown) and purified protein was used for single-particle cryo-EM (Supplementary Fig. 1), and two different datasets were collected from different preparations. The data rendered density maps at 3.5 and $3.6 \AA$ resolution, which were of sufficient quality for the de novo building and refinement of an atomic model of the protein (Supplementary Fig. 1 and Supplementary Table 1).

The EBV portal shows a ring-like structure, consisting of 12 subunits. In contrast to bacteriophage portals, where particles have been reported as 12 -mers and 13 -mers ${ }^{10-14}$, no indication of an oligomerization state other than 12 was detected in the micrographs or $2 \mathrm{D}$ averages. The particle of the EBV portal has a mushroom-like shape, with a maximum external diameter of 140 $\AA$ at the "cap" and $62 \AA$ at the "stem", while the height is $116 \AA$ (Fig. 1). There is an internal conical channel which narrows from the "cap" of the mushroom to its "stem", with a diameter ranging from 75 to $31 \AA$, respectively (Fig. 2). The wide "cap" points to the interior of the capsid, while the narrow "stem" makes the channel to the exterior (see below). The structure comprises three domains. There are marked differences from the bacteriophage portals, however, for comparison purposes we follow their nomenclature and name the domains wing, stem, and crown (Fig. 1 , Supplementary Movie 1). The wing or central domain connects the crown to the stem, and contains eight $\beta$-strands-three of them relatively short-and three short $\alpha$-helices (Fig. 1). There are two nearly perpendicular $\beta$-sheets, each of them composed of three antiparallel $\beta$-strands, forming an SH3-like structure, similar to that found in portal protein gp10 of $\phi 29^{10}$. The Nterminus is also located in this domain, although the first 16 residues are not resolved in our maps. The wing is the outermost domain, however, the EBV portal wing protrudes less than the corresponding structure in bacteriophage portals, since the wide part of the crown cone has about the same external diameter. The stem region comprises two antiparallel $\alpha$-helices with different length, $\alpha 8$ and $\alpha 15$ (Fig. 1), which shape the walls of the channel, a feature conserved among all portal proteins. They are tilted relative to the tunnel axis by $45^{\circ}$ and $30^{\circ}$, respectively. After the two helices, there are three $\beta$-strands and a further short helix (a14) that embraces the neighboring monomer and is positioned almost perpendicular to a8 (Fig. 1). After the $\beta$-strands there are five predicted helices that are not assigned in the current structure (from residue 288 to 433) because the density is too poor to allocate their position, which reflects the flexibility of the distal tip region of the EBV portal. Based on the weak density in this area, we can, however, infer that these helices make a longer channel after the $\beta$-tunnel, and are flexible in order to adjust to the fivefold symmetry of the portal vertex protein (see below).

The channel valve of EBV portal. The crown is the most variable region in bacteriophage portal proteins. This region is absent in phage $\$ 29$ portal $^{10,14}$, while in P22 portal it has a long $\alpha$-barrel shaped structure (Supplementary Fig. 2) ${ }^{12}$. In the EBV portal, the crown is larger than in most bacteriophage portals and includes at least eight $a$-helices of varying size. There is a clear separation between the wing and the crown with no structured secondary structure elements crossing between the two domains (except for the channel valve, see below), however, the deep cleft between the domains observed in some bacteriophage portals, is not present (Fig. 1a-c and S2). The C-terminus of the protein should be located at the crown, although the density of the last 40 residues is not clearly visible. In the monomer, the crown domain is tilted about $66^{\circ}$ relative to the stem domain, with the wing acting as a hinge (Fig. 1c). The channel valve ${ }^{20}$ (also termed tunnel loop) is located at the crown and the wing interface, at the channel interior. In most bacteriophage portals, the channel valve is formed by a long $\alpha$-helix which protrudes from the wing towards 
a
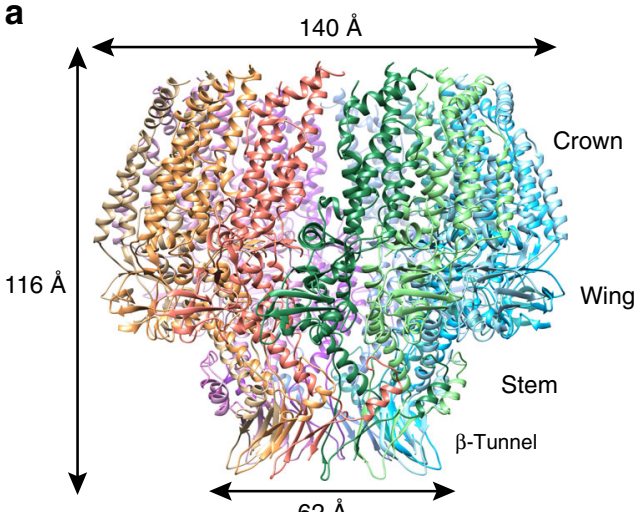

b

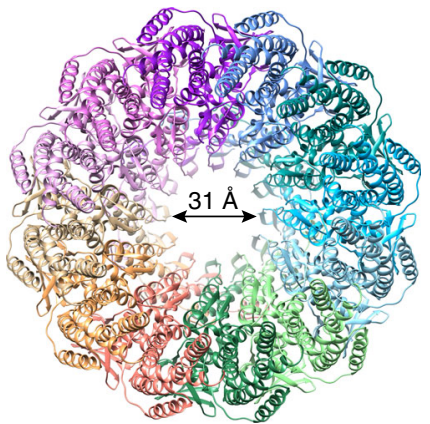

C

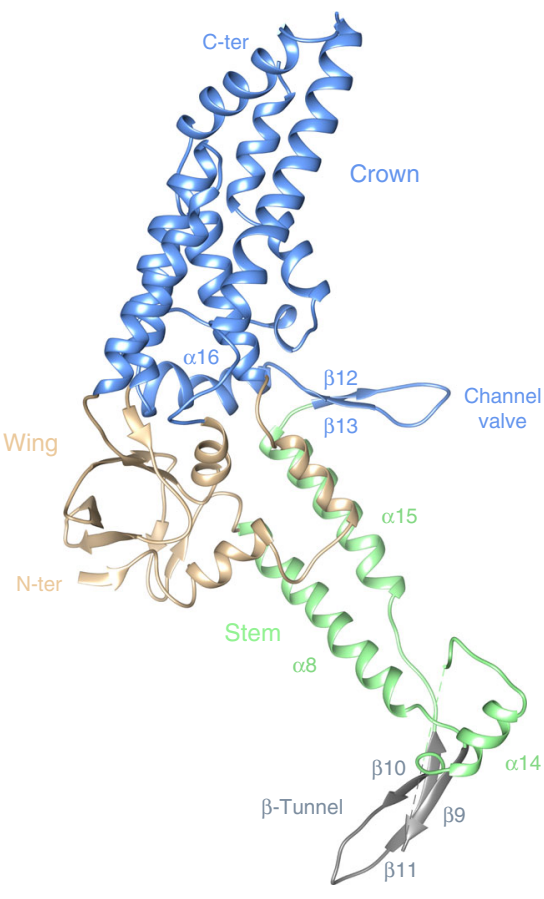

Fig. 1 Structural characterization of EBV portal. Ribbon representation of the EBV portal cryo-EM structure, indicating the dimensions of the particle, and diameter of the inner channel. a Lateral view. b Axial view. c Ribbon representation of EBV portal monomer colored by regions: crown (blue), wing (sand), stem (light green), and $\beta$-hairpin channel valve (gray). The channel valve and secondary elements relevant for protein-protein interactions are also indicated

the channel and ends in the tunnel loop, which is between 10 and 26 residues long ${ }^{11-13}$ and connects back to the stem. This helix and loop have been proposed to undergo a movement that plays a crucial role during DNA packaging ${ }^{11,20}$. However, in the EBV portal, the equivalent helix, a16, is shorter, forms part of the crown, not of the wing, and does not protrude into the channel. Instead, $\alpha 16$ continues in the channel as a $\beta$-strand that forms a $\beta$-ribbon, with the tunnel loop at its tip. The $\beta$-ribbon valve thus connects $\alpha 16$ with $\alpha 15$, the latter being one of the two long helices of the stem. This loop of the $\beta$-ribbon is not well defined, showing weak density for the six residues at the tip, indicating the flexibility of this region, a feature also observed in bacteriophage portals. Without these six residues, the aperture inside the channel at this level would be around $36 \AA$. However, when residues are modeled into the weak density, the aperture is reduced to about the diameter of the B-form DNA. This structural feature clearly corresponds to the valve observed in bacteriophage portal structures ${ }^{20}$ and most probably plays a similar role in securing the DNA inside the capsid. It is interesting to note the position of helix $\alpha 16$, which is oriented perpendicular to the channel axis and between monomers. Inter-monomer contacts at the crown are scarce, giving the impression that there is a cleft between the protomers (Fig. 1a, Supplementary Fig. 1, and Supplementary Movie 1).

A $\beta$-tunnel forms a second narrow section. In the EBV portal, in addition to the channel valve, another narrow section in the channel is located at end of the stem, at the level of strand $\beta 11$ (Fig. 2). This leaves an aperture of $31 \AA$, which would allow easy passage of B-DNA. This constriction is quite different from the channel valve, since it extends for about $17 \AA$ along the channel, forming a tunnel. Furthermore, it is a fixed structure with no indication of mobility. Indeed, this cylindrical structure is formed by the inter-monomer assembly of 12 three-stranded $\beta$-sheets with their planes radially disposed around the channel in the dodecamer (Fig. 2a). Two $\beta$-strands-parallel to each otherbelong to one monomer, while the third $\beta$-strand, antiparallel, belongs to the adjacent subunit, thus building a strong interaction between monomers. A similar arrangement has been found in bacteriophage portal proteins ${ }^{10,11,13}$, however, the EBV portal differs in the disposition of these $\beta$-sheets, since their edges face the interior of the channel, forming the stiff cylindrical structure that we name the $\beta$-tunnel (Fig. 2). This structure, which includes the hydrophobic side chain of Phe 470 at the channel surface, is expected to play a role in guiding DNA passage, but there is no indication for it being a gate or a valve with an open/close motion.

Adjacent protomers of the EBV portal dodecamer have a large contact area of $4050 \AA^{2}$. The oligomer is stabilized by a complex network of interactions, located mostly at the stem domain, where each protomer interacts with up to four distinct neighboring protomers. In addition to the inter-protomer interactions involved in the assembly of the $\beta$-tunnel described above, helix $\alpha 8$ also contributes to dodecamer stabilization. The residues of this helix interact with three distinct monomers, in such a way that residues of helix $\alpha 8$ of monomer A interact with residues in the loop before helix a 14 of monomers $B$ and $C$, as well as residues of helix a 8 of monomer L. There are also some complementary charged areas buried in the interacting surface between monomers in the wing and crown regions, which establish salt bridges between them (Fig. 2b). A putative disulfide bridge between Cys166 and Cys254 has been described in pUL6 from HSV-1. This may contribute to the stabilization of HSV-1 portal, since the presence of dithiotreitol results in the disruption of the dodecameric rings, and the mutation of those residues 


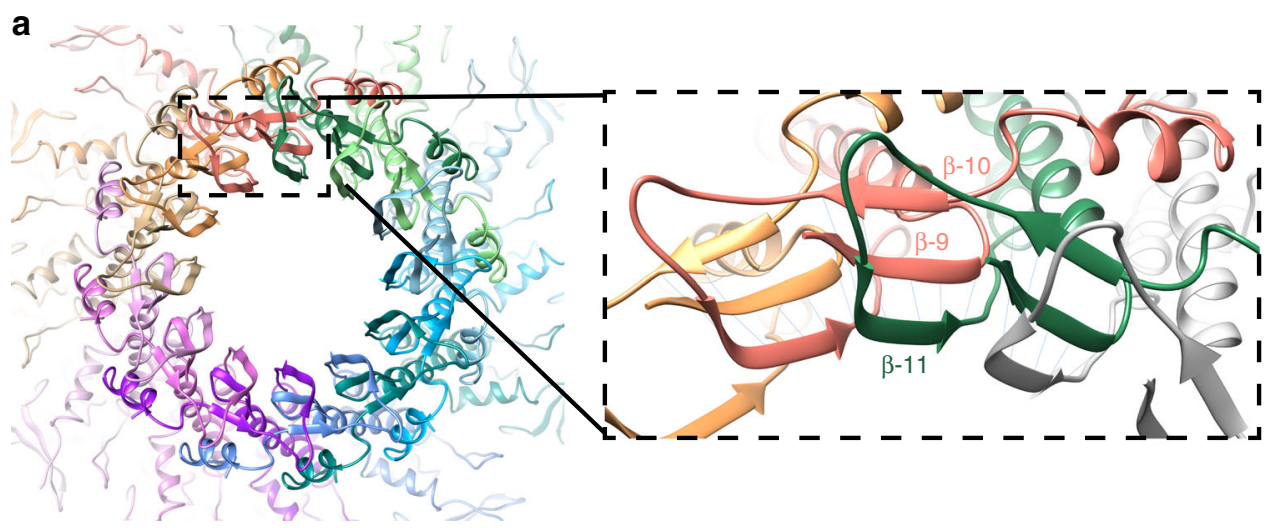

b

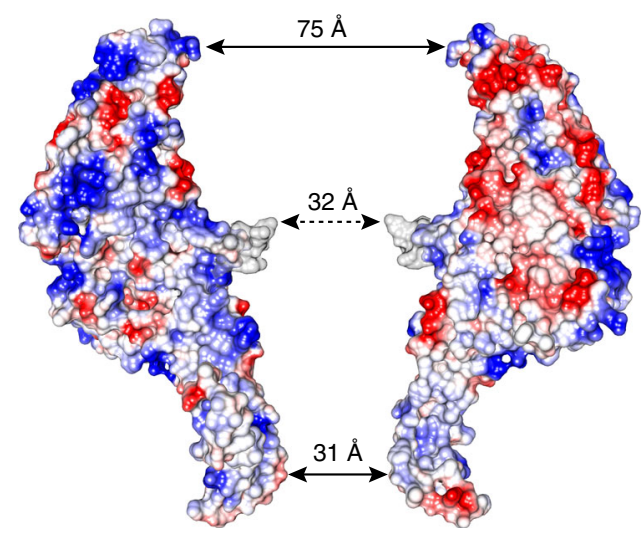

Fig. 2 Protein-protein interactions in the EBV portal oligomer. a Axial view of EBV portal protein at the $\beta$-tunnel region (left), and a close-up view of protomer-protomer $\beta$-sheet formation (right) with $\beta$-strands 9, 10 of one protomer and 11, from an adjacent protomer. $\mathbf{b}$ Electrostatic potential of two opposing monomers within EBV portal dodecamer. Blue represents $10 \mathrm{kcal} /(\mathrm{mol} \cdot \mathrm{e})$ positive potential, while red represents $-10 \mathrm{kcal} /(\mathrm{mol} \cdot e)$ negative potential. Indication of the distance between residues in the central part of the channel at different levels is included. Gray shows the six residues of the tunnel loop with weak density
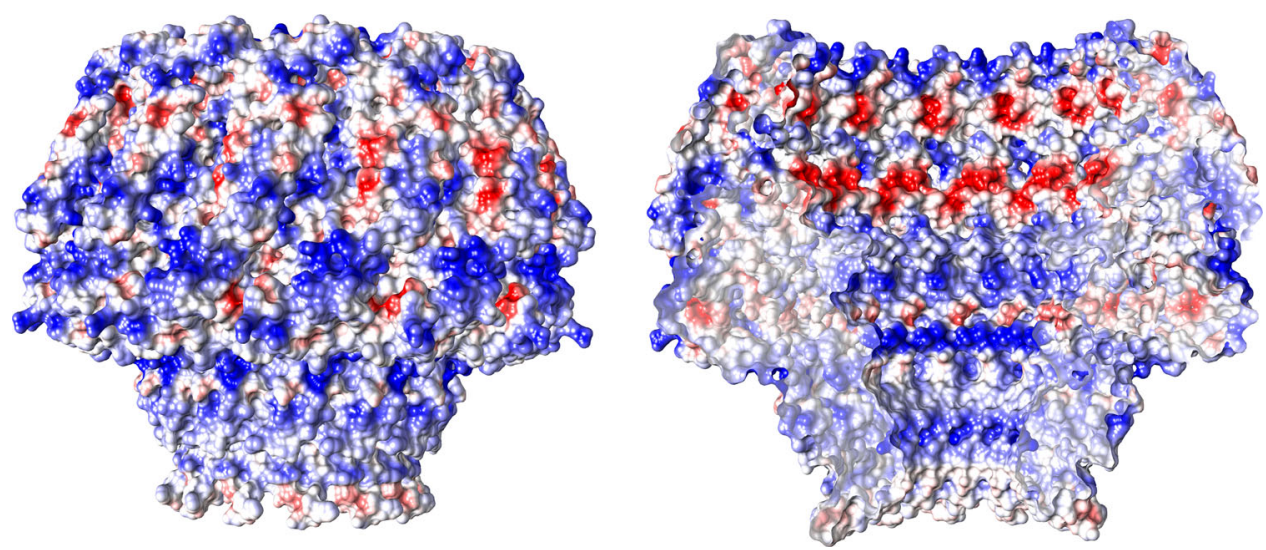

Fig. 3 EBV portal electrostatic potential. a Electrostatic potential on the external (left) and inner (right) surfaces of EBV portal dodecamer. Blue represents $10 \mathrm{kcal} /(\mathrm{mol} \cdot \mathrm{e})$ positive potential, while red represents $-10 \mathrm{kcal} /(\mathrm{mol} \cdot \mathrm{e})$ negative potential

produced capsids with a reduced level of pUL6 ${ }^{21}$. These Cys residues are not conserved in EBV and we did not find any other disulfide bridge in the current structure.

Interaction of EBV portal with capsid proteins. The electrostatic potential of the EBV portal is distinct from that of the bacteriophage portal proteins (Fig. 3), the external surface of the EBV portal is mainly positively charged, while the phage portals are mostly negative ${ }^{13}$. This difference may have implications for the interactions established between the EBV portal and the rest of the proteins of the capsid and the packaged DNA. Figure 4 shows our EBV portal structure fitted in the portal vertex of the HSV - $18 \AA$ density with the atomic coordinates of the HSV-2 capsid also fitted (with a penton of the major capsid protein removed at the pentameric vertex to accommodate the portal $)^{22-24}$. The good fit of the portal in the density, with a correlation coefficient of 0.7803 -even though the density was fivefold averaged instead of 12 -fold as for 


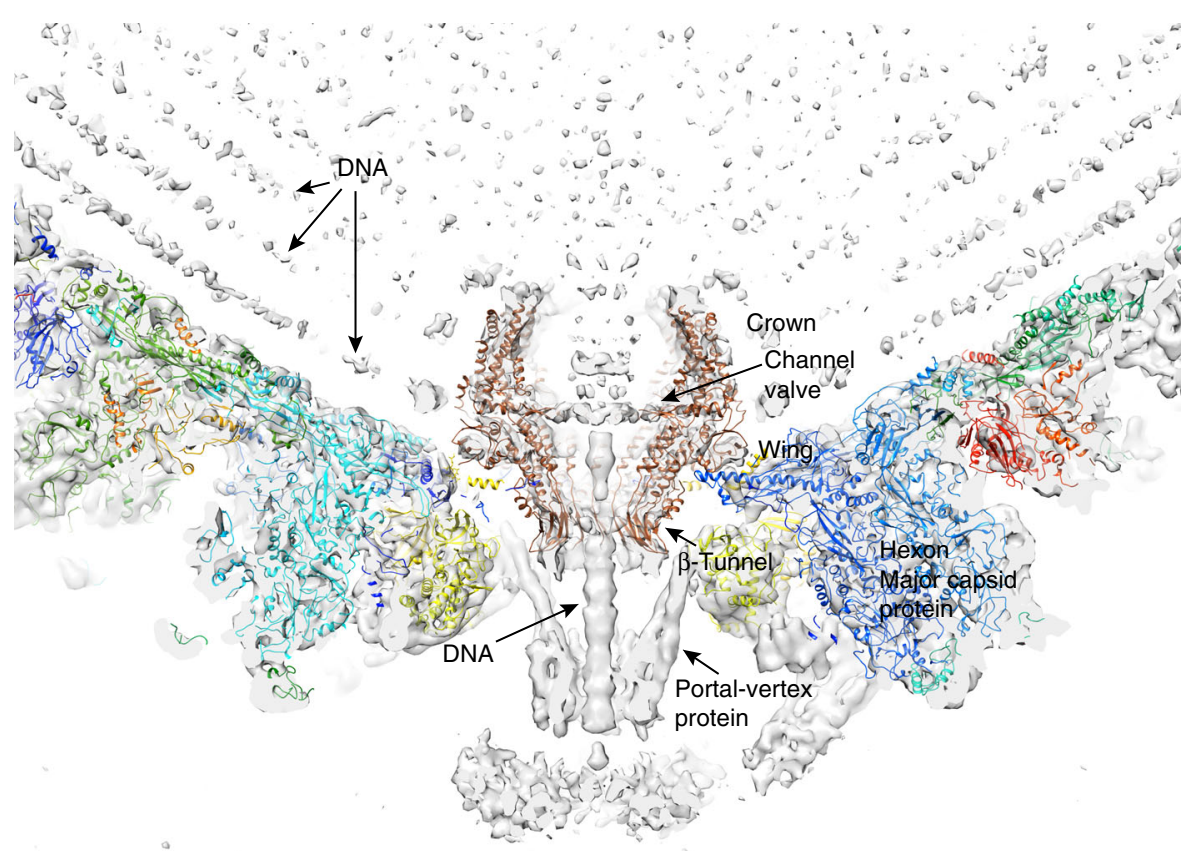

Fig. 4 EBV portal fitting in the HSV-1 capsid portal vertex map. The EBV portal structure (brown-sienna, this work) and the capsid components of HSV-2 (rainbow colors, PDB 5ZZ822, 24) were docked into the portal vertex density of HSV-1 capsid, at the unique portal vertex (gray, EMD 434723) with Chimera ${ }^{44}$. The relevant domains of EBV portal protein, the interacting capsid proteins, as well as the viral DNA are indicated

the portal protein-indicates that the position of the portal with respect to the capsid is correct. The figure clearly indicates that the portal is rather internal, with its crown fully inside the capsid. The portal is held mainly through its wing, which interacts with the major capsid protein of the adjacent hexons, and also with an unknown helical pentameric protein at the end of the so-called clip in the bacteriophage portals. It is interesting that the wing has a SH3-like domain, a structure which mediates protein-protein interactions in other systems $\mathrm{s}^{25}$. The three positively charged rings of the crown suggest that they play a role in the interaction with the packaged DNA phosphates (Fig. 3), since they roughly coincide with layers of the DNA genome. The density of the portal vertex reveals that the $\beta$-ribbon channel valve is well ordered and closed, with the internal DNA pressed against the valve. An open question is whether the valve closes due to a movement of the crown, as a result of the pressure of the DNA once the capsid is full-a movement that could be transmitted to $\alpha 16$ and its contiguous $\beta$ ribbon to close the valve. The loose interaction between protomers at the crown indicates that the crown might be compressible.

This work describes a high-resolution atomic structure of a herpesvirus portal particle. An alignment of EBV portal sequence against the sequences of all the other portal proteins of herpesviruses shows $23-25 \%$ sequence identity, except for pORF43 from Kaposi's sarcoma-associated herpesvirus, which shows $51 \%$ sequence identity, since it belongs to the same subfamily of gammaherpesvirinae. Among the most conserved regions between all herpesvirus portals are those involved in protein-protein interactions, such as $\alpha 8$ helix or $\beta 11$ strands, and the channel valve/tunnel loop region.

The drugs currently licensed for the treatment of herpesvirus infections target the viral DNA polymerase. They show poor efficacy because of the appearance of resistant viral strains or serious side effects after a prolonged treatment ${ }^{26-28}$. In the case of EBV infection, the situation is even more critical, because there is no specific treatment for this severe disease. Given the key role of portal proteins in herpesvirus DNA packaging and release, as well as during capsid formation, their atomic structures will be extremely valuable for the rational design of compounds targeting their function. A number of herpes portal inhibitors have been reported $^{9}$; however, their modes of action and binding are unknown, hindering their further development. The structure presented here paves the way to prepare and solve inhibitorportal binary complexes, which in turn will accelerate their optimization and allow the design of new highly specific antivirals.

\section{Methods}

Cloning of bbrf1 gene. The bbrf1 gene, which codes for the EBV portal protein, was amplified by PCR from genomic DNA purchased from The National Collection of Pathogenic Viruses (NCPV) (Epstein-Barr virus \#634), using the oligonucleotides (the sequence annealing with bbrf1 is indicated in bold) bbrf1_EBV_pPEU10_F: 5'-AAGTTCTGTTTCAGGGCCCGATGTTCAACAT GAACGTGGACGA-3' and bbrf1_EBV_pPEU10_R: 5'-ATGGTCTAGAAA GCTTTAACTTCCAGCACCAGGCGGG-3'. The 1842-bp PCR-amplified fragment was cloned in KpnI-HindIII digested pPEU11 vector (provided by the Protein Expression Facility, at the Institute for Research in Biomedicine (IRB-Barcelona)) by an In-Fusion ${ }^{\circledR}$ reaction (Clontech). The resulting construct, pCMS1017, was checked by sequencing and used for the expression of the fusion protein His-ZpBBRF1 in Baculovirus.

Purification of pBBRF1 from recombinant baculovirus. Recombinant baculovirus was produced following the instructions of baculoCOMPLETE ${ }^{\mathrm{TM}}$ ALL-IN-ONE Baculovirus Protein Expression kit from Oxford Expression Technologies. The protein expression conditions were optimized by infection of a monolayer of Spodoptera frugiperda Sf9 cells with the produced baculovirus. The cells were harvested after $72 \mathrm{~h}$ of incubation at $28^{\circ} \mathrm{C}$, when the expression of His-Z-pBBRF1 was optimal, by centrifugation of the media at $3000 \times g$ for $20 \mathrm{~min}$ at $4{ }^{\circ} \mathrm{C}$. The cell pellet was lysed by pipetting in lysis buffer $(20 \mathrm{mM}$ Tris- $\mathrm{HCl} \mathrm{pH} 8.0,500 \mathrm{mM}$ $\mathrm{NaCl}, 20 \mathrm{mM}$ imidazole, $5 \mathrm{mM} \beta$-mercaptoethanol, and $0.2 \% \mathrm{n}$-dodecyl-betamaltoside (DDM) (w/v)), and passing it through a cell disruptor (Constant Systems Ltd) at $25 \mathrm{kpsi}$. Following centrifugation at $40,000 \times g$ for $20 \mathrm{~min}$ at $4{ }^{\circ} \mathrm{C}$, the sample was filtered and loaded on a HisTrap ${ }^{\mathrm{TM}}$ HP 5-ml (GE Healthcare Life Sciences). His-Z-pBBRF1 was eluted from the column with a gradient between 20 and 500 $\mathrm{mM}$ imidazole (20 mM Tris- $\mathrm{HCl} \mathrm{pH} 8.0,500 \mathrm{mM} \mathrm{NaCl}, 5 \mathrm{mM} \beta$-mercaptoethanol, and $0.05 \% \mathrm{n}$-dodecyl-beta-maltoside (DDM) (w/v)). The fractions containing HisZ-pBBRF1 were pooled and loaded on a HiTrap ${ }^{\text {TM }}$ Heparin HP 1-ml (GE Healthcare Life Sciences) and eluted with a gradient between $150 \mathrm{mM}$ and $1.5 \mathrm{M}$ $\mathrm{NaCl}$ of heparin buffer $(50 \mathrm{mM}$ Tris- $\mathrm{HCl} \mathrm{pH}$ 8.0, $20 \mathrm{mM} \beta$-mercaptoethanol, $0.05 \%$ DDM (w/v), $1 \mathrm{mM}$ EDTA). Fractions enriched with His-Z-pBBRF1 were then loaded on a HiPrep 16/60 Sephacryl S-400 HR (GE Healthcare Life Sciences), equilibrated with heparin buffer with $500 \mathrm{mM} \mathrm{NaCl}$, and afterwards on a Superose 6 10/300 GL (GE Healthcare Life Sciences). The eluted fractions were analyzed by 
gel electrophoresis and concentrated with an Amicon Ultra 100K. At this stage, the protein was ready for further analysis and characterization.

Cryo-EM data collection. Cryo-EM samples were prepared in Quantifoil R2/2 copper grids coated with a thin carbon film, which was made in house. The grids were glow discharged for $60 \mathrm{~s}$ at $25 \mathrm{~mA}$ using an Emitech Glow Discharge machine, before applying $3 \mu \mathrm{l}$ of purified His-Z-pBBRF1 at a concentration of $0.1 \mathrm{mg} / \mathrm{ml}$. The sample was incubated for $2 \mathrm{~min}$ at room temperature on the grid, before being blotted and applying another $3 \mu \mathrm{l}$ of heparin buffer with $500 \mathrm{mM} \mathrm{NaCl}$, without $\mathrm{DDM}$. This procedure was repeated twice, before the final blotting of the sample on a VitroBot Mark IV (FEI) system at $20^{\circ} \mathrm{C}$ and $95 \%$ humidity, for $3.5 \mathrm{~s}$, blotting force: 0 , and plunge-freezing it into liquid ethane.

Data were collected on a Titan Krios (FEI) transmission electron microscope at $300 \mathrm{keV}$, using a Gatan Quantum energy filter and a K2 Summit direct detector, at the Electron Bio-Imaging Centre (eBIC) at Diamond Light Source (Oxford, UK) and The Netherlands Centre for Electron Nanoscopy (NeCEN) at the Universiteit Leiden (Leiden, The Netherlands). In total, 2172 and 5431 movies were collected at eBIC and at NeCEN, at a 1.06 and $1.1 \AA$ pixel size, respectively, within a defocus range from -1.0 to $-3.0 \mu \mathrm{m}$ and at a dose rate of $6.7 \mathrm{e}^{-} / \AA^{2} / \mathrm{s}$, in Super-Resolution mode for eBIC.

Cryo-EM images processing and map calculation. Cryo-EM data processing was performed using both the Scipion software framework ${ }^{29}$ and RELION 3.030. Dosefractionated image stacks were motion-corrected and dose-weighted using MotionCor $2^{31}$. Contrast transfer function (CTF) was estimated using the CTFfind4 program $^{32}$. Particles were picked using xmipp $3^{33}$, RELION auto-picking, and Gautomatch within the Scipion software framework ${ }^{29}$. Extracted particles were classified using RELION 2D and 3D, after building an initial volume with Ransac ${ }^{33}$ (NeCEN data) or RELION (eBIC data), applying C12 symmetry to the model. Final volumes were obtained using RELION Auto-refine with 73,395 particles for the model at $3.5 \AA$ resolution (NeCEN data) and with 25,788 particles for the model at $3.6 \AA$ (eBIC data). The structure resolution was estimated from RELION FSC curves with the 0.143 cutoff criterion, and local resolution was computed with MonoRes ${ }^{34}$

Structure solution, model building, and coordinate refinement. A partial model of EBV portal was obtained after providing the sequence of pBBRF1 and the cryoEM map to Buccaneer ${ }^{35}$, within CCP-EM framework ${ }^{36,37}$. The remaining structure was built ab initio in $\operatorname{Coot}^{38}$, considering the secondary structure prediction obtained from PSIPRED ${ }^{39,40}$ as a guide. PHENIX real space refinement ${ }^{41}$ and REFMAC $5^{42}$ within the CCP-EM suite for cryo-EM data were used to refine the models. The models were validated using MolProbity ${ }^{43}$. Both models are very similar, with an r.m.s.d. of $0.88 \AA$, although the eBIC data map was better defined in some peripheral areas, and helix $\alpha 6$ could be assigned in this case. Clear density, although weak, was also present at the loop of the $\beta$-hairpin tunnel valve in this map.

Reporting summary. Further information on research design is available in the Nature Research Reporting Summary linked to this article.

\section{Data availability}

The electron microscopy maps have been deposited in the Electron Microscopy Data Bank (EMDB) and Protein Data Bank with accession codes EMD-10010 and EMD10011, 6RVR, and 6RVS, respectively. All other relevant data are available from the authors upon request.

Received: 7 June 2019 Accepted: 25 July 2019

Published online: 29 August 2019

\section{References}

1. Grinde, B. Herpesviruses: latency and reactivation-viral strategies and hos response. J. Oral. Microbiol. 5, 22766 (2013).

2. Kutok, J. L. \& Wang, F. Spectrum of Epstein-Barr virus-associated diseases. Annu Rev. Pathol. 1, 375-404 (2006).

3. Hammerschmidt, W. \& Sugden, B. Epstein-Barr virus sustains Burkitt's lymphomas and Hodgkin's disease. Trends Mol. Med 10, 331-336 (2004).

4. Baker, M. L., Jiang, W., Rixon, F. J. \& Chiu, W. Common ancestry of herpesviruses and tailed DNA bacteriophages. J. Virol. 79, 14967-14970 (2005).

5. Bamford, D. H., Grimes, J. M. \& Stuart, D. I. What does structure tell us about virus evolution? Curr. Opin. Struct. Biol. 15, 655-663 (2005)

6. Rixon, F. J. \& Schmid, M. F. Structural similarities in DNA packaging and delivery apparatuses in Herpesvirus and dsDNA bacteriophages. Curr. Opin. Virol. 5, 105-110 (2014).
7. Brown, J. C. \& Newcomb, W. W. Herpesvirus capsid assembly: insights from structural analysis. Curr. Opin. Virol. 1, 142-149 (2011).

8. Cuervo, A. \& Carrascosa, J. L. Viral connectors for DNA encapsulation. Curr. Opin. Biotechnol. 23, 529-536 (2012).

9. Kornfeind, E. M. \& Visalli, R. J. Human herpesvirus portal proteins: structure, function, and antiviral prospects. Rev. Med Virol. 28, e1972 (2018).

10. Guasch, A. et al. Detailed architecture of a DNA translocating machine: the high-resolution structure of the bacteriophage phi29 connector particle. J. Mol. Biol. 315, 663-676 (2002).

11. Lebedev, A. A. et al. Structural framework for DNA translocation via the viral portal protein. EMBO J. 26, 1984-1994 (2007)

12. Olia, A. S., Prevelige, P. E. Jr, Johnson, J. E. \& Cingolani, G. Threedimensional structure of a viral genome-delivery portal vertex. Nat. Struct. Mol. Biol. 18, 597-603 (2011).

13. Sun, L. et al. Cryo-EM structure of the bacteriophage T4 portal protein assembly at near-atomic resolution. Nat. Commun. 6, 7548 (2015).

14. Simpson, A. A. et al. Structure of the bacteriophage phi29 DNA packaging motor. Nature 408, 745-750 (2000).

15. Holzenburg, A., Dittmer, A. \& Bogner, E. Assembly of monomeric human cytomegalovirus pUL104 into portal structures. J. Gen. Virol. 90, 2381-2385 (2009).

16. Howard, A. J., Sherman, D. M., Visalli, M. A., Burnside, D. M. \& Visalli, R. J. The Varicella-zoster virus ORF54 gene product encodes the capsid portal protein, pORF54. Virus Res. 167, 102-105 (2012).

17. Trus, B. L. et al. Structure and polymorphism of the UL6 portal protein of herpes simplex virus type 1. J. Virol. 78, 12668-12671 (2004).

18. Visalli, R. J., Schwartz, A. M., Patel, S. \& Visalli, M. A. Identification of the Epstein Barr Virus portal. Virology 529, 152-159 (2019).

19. Liu, Y. T., Jih, J., Dai, X., Bi, G. Q. \& Zhou, Z. H. Cryo-EM structures of herpes simplex virus type 1 portal vertex and packaged genome. Nature 570, 257-261 (2019).

20. Cuervo, A. et al. Structures of T7 bacteriophage portal and tail suggest a viral DNA retention and ejection mechanism. Nat Commun. https://doi.org/ 10.1038/s41467-019-11705-9 (2019).

21. Albright, B. S., Nellissery, J., Szczepaniak, R. \& Weller, S. K. Disulfide bond formation in the herpes simplex virus 1 UL6 protein is required for portal ring formation and genome encapsidation. J. Virol. 85, 8616-8624 (2011).

22. Dai, X. \& Zhou, Z. H. Structure of the herpes simplex virus 1 capsid with associated tegument protein complexes. Science 360, eaao7298 (2018).

23. McElwee, M., Vijayakrishnan, S., Rixon, F. \& Bhella, D. Structure of the herpes simplex virus portal-vertex. PLoS Biol. 16, e2006191 (2018)

24. Yuan, S. et al. Cryo-EM structure of a herpesvirus capsid at 3.1 A. Science 360, eaao7283 (2018).

25. Kurochkina, N. \& Guha, U. SH3 domains: modules of protein-protein interactions. Biophys. Rev. 5, 29-39 (2013).

26. Andrei, G. \& Snoeck, R. Herpes simplex virus drug-resistance: new mutations and insights. Curr. Opin. Infect. Dis. 26, 551-560 (2013).

27. Piret, J. \& Boivin, G. Antiviral resistance in herpes simplex virus and varicellazoster virus infections: diagnosis and management. Curr. Opin. Infect. Dis. 29, 654-662 (2016).

28. Schreiber, A. et al. Antiviral treatment of cytomegalovirus infection and resistant strains. Expert Opin. Pharm. 10, 191-209 (2009).

29. de la Rosa-Trevin, J. M. et al. Scipion: a software framework toward integration, reproducibility and validation in 3D electron microscopy. $J$. Struct. Biol. 195, 93-99 (2016).

30. Zivanov, J. et al. New tools for automated high-resolution cryo-EM structure determination in RELION-3. Elife 7, e42166 (2018).

31. Zheng, S. Q. et al. MotionCor2: anisotropic correction of beam-induced motion for improved cryo-electron microscopy. Nat. Methods 14, 331-332 (2017).

32. Rohou, A. \& Grigorieff, N. CTFFIND4: Fast and accurate defocus estimation from electron micrographs. J. Struct. Biol. 192, 216-221 (2015).

33. de la Rosa-Trevin, J. M. et al. Xmipp 3.0: an improved software suite for image processing in electron microscopy. J. Struct. Biol. 184, 321-328 (2013)

34. Vilas, J. L. et al. MonoRes: automatic and accurate estimation of local resolution for electron microscopy maps. Structure 26, 337-344 e4 (2018).

35. Cowtan, K. The Buccaneer software for automated model building. 1. Tracing protein chains. Acta Crystallogr D. Biol. Crystallogr. 62, 1002-1011 (2006).

36. Burnley, T., Palmer, C. M. \& Winn, M. Recent developments in the CCP-EM software suite. Acta Crystallogr D. Struct. Biol. 73, 469-477 (2017).

37. Nicholls, R. A., Tykac, M., Kovalevskiy, O. \& Murshudov, G. N. Current approaches for the fitting and refinement of atomic models into cryo-EM maps using CCP-EM. Acta Crystallogr D. Struct. Biol. 74, 492-505 (2018).

38. Emsley, P. \& Cowtan, K. Coot: model-building tools for molecular graphics. Acta Crystallogr D. Biol. Crystallogr. 60, 2126-2132 (2004).

39. Buchan, D. W., Minneci, F., Nugent, T. C., Bryson, K. \& Jones, D. T. Scalable web services for the PSIPRED Protein Analysis Workbench. Nucleic Acids Res. 41, W349-W357 (2013). 
40. Jones, D. T. Protein secondary structure prediction based on position-specific scoring matrices. J. Mol. Biol. 292, 195-202 (1999).

41. Afonine, P. V. et al. Real-space refinement in PHENIX for cryo-EM and crystallography. Acta Crystallogr D. Struct. Biol. 74, 531-544 (2018).

42. Murshudov, G. N. et al. REFMAC5 for the refinement of macromolecular crystal structures. Acta Crystallogr D. Biol. Crystallogr. 67, 355-367 (2011).

43. Williams, C. J. et al. MolProbity: more and better reference data for improved all-atom structure validation. Protein Sci. 27, 293-315 (2018).

44. Pettersen, E. F. et al. UCSF Chimera-a visualization system for exploratory research and analysis. J. Comput Chem. 25, 1605-1612 (2004).

\section{Acknowledgements}

This work was partly supported by the Ministry of Science, Innovation and Universities of Spain, grants BFU2014-53550-P and BFU2017-83720-P (to M.C.), BFU 2014-54181 (to J.L.C.), and contracts SEV-2013-0347 (to A.C.), and RYC-2011-09071 (to C.M.). We acknowledge institutional funding through the Spanish Government Centres and Units of Excellence, Severo Ochoa and María de Maeztu awards, to IRB-Barcelona (SEV-20150500) and IBMB Structural Biology Unit (MDM-2014-0435), respectively; and from the CERCA Programme of the Catalan Government to IRB-Barcelona. This work has also been supported by iNEXT, grant number 653706, funded by the Horizon 2020 programme of the European Commission, which allowed us access to transmission Titan Krios electron microscopes at the Electron Bio-Imaging Centre (eBIC) at Diamond Light Source (Oxford, UK) and the Netherlands Centre for Electron Nanoscopy (NeCEN) at the Universiteit Leiden (Leiden, The Netherlands). The authors also acknowledge the use and support of the Centro Nacional de Biotecnología-Centro de Investigaciones Biológicas (CNB-CIB) Cryo-EM facility (CSIC) in Madrid (Spain), and resources of InstructERIC.

\section{Author contributions}

M.C. conceived and coordinated the project. C.M. prepared the proteins. A.C. and C.M. prepared the cryo-EM grids. C.M., M.F.-F., and M.C. collected the cryo-EM data. C.M., M.F.-F., D.Z., A.C., and M.C. processed the cryo-EM data. C.M., M.F.-F., D.Z., and M.C. solved the cryo-EM structures. C.M., M.F.-F., A.C., J.C.L., D.I.S., and M.C. analyzed and discussed the results. C.M., M.F.-F., and M.C. wrote the paper with contributions from all authors.

\section{Additional information}

Supplementary Information accompanies this paper at https://doi.org/10.1038/s41467019-11706-8.

Competing interests: The authors declare no competing interests.

Reprints and permission information is available online at http://npg.nature.com/ reprintsandpermissions/

Peer review information: Nature Communications thanks David Bhella, Gino Cingolani for their contribution to the peer review of this work.

Publisher's note: Springer Nature remains neutral with regard to jurisdictional claims in published maps and institutional affiliations.

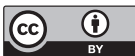

Open Access This article is licensed under a Creative Commons Attribution 4.0 International License, which permits use, sharing, adaptation, distribution and reproduction in any medium or format, as long as you give appropriate credit to the original author(s) and the source, provide a link to the Creative Commons license, and indicate if changes were made. The images or other third party material in this article are included in the article's Creative Commons license, unless indicated otherwise in a credit line to the material. If material is not included in the article's Creative Commons license and your intended use is not permitted by statutory regulation or exceeds the permitted use, you will need to obtain permission directly from the copyright holder. To view a copy of this license, visit http://creativecommons.org/ licenses/by/4.0/

(C) The Author(s) 2019 